Mon. Not. R. Astron. Soc. 000, 1 13 (2016) Printed 4 October $2018 \quad$ (MN LATEX style file v2.2)

\title{
The core-degenerate scenario for the progenitors of type Ia supernovae
}

\author{
B. Wang, ${ }^{1,2,3 \star}$ W.-H. Zhou, ${ }^{2,4}$ Z.-Y. Zuo, ${ }^{5}$ Y.-B. Li, ${ }^{6}$ X. Luo, ${ }^{7}$ J.-J. Zhang, ${ }^{1,2}$ \\ D.-D. Liu, ${ }^{1,2,3}$ and C.-Y. Wu ${ }^{1,2,3}$ \\ ${ }^{1}$ Yunnan Observatories, Chinese Academy of Sciences, Kunming 650216, China \\ ${ }^{2}$ Key Laboratory for the Structure and Evolution of Celestial Objects, Chinese Academy of Sciences, Kunming 650216, China \\ ${ }^{3}$ University of Chinese Academy of Sciences, Beijing 100049, China \\ ${ }^{4}$ Yunnan Minzu University, Kunming 650031, China \\ ${ }^{5}$ School of Science, Xi'an Jiaotong University, Xi'an 710049, China \\ ${ }^{6}$ Key Laboratory of Optical Astronomy, National Astronomical Observatories, Chinese Academy of Sciences, Beijing 100012, China \\ ${ }^{7}$ Department of Astronomy, Beijing Normal University, Beijing 100875, China
}

Accepted. Received

\begin{abstract}
The origin of the progenitors of type Ia supernovae (SNe Ia) is still uncertain. The core-degenerate (CD) scenario has been proposed as an alternative way for the production of SNe Ia. In this scenario, SNe Ia are formed at the final stage of common-envelope evolution from a merger of a carbon-oxygen white dwarf (CO WD) with the CO core of an asymptotic giant branch companion. However, the birthrates of SNe Ia from this scenario are still not well determined. In this work, we performed a detailed investigation on the CD scenario based on a binary population synthesis approach. The SN Ia delay times from this scenario are basically in the range of $90 \mathrm{Myr}-2500 \mathrm{Myr}$, mainly contributing to the observed SNe Ia with short and intermediate delay times although this scenario can also produce some old SNe Ia. Meanwhile, our work indicates that the Galactic birthrates of SNe Ia from this scenario are no more than $20 \%$ of total SNe Ia due to more careful treatment of mass transfer. Although the SN Ia birthrates in the present work are lower than those in Ilkov \& Soker, the CD scenario cannot be ruled out as a viable mechanism for the formation of SNe Ia. Especially, SNe Ia with circumstellar material from this scenario contribute to $0.7-10 \%$ of total SNe Ia, which means that the CD scenario can reproduce the observed birthrates of SNe Ia like PTF $11 \mathrm{kx}$. We also found that SNe Ia happen systemically earlier for a high value of metallicity and their birthrates increase with metallicity.
\end{abstract}

Key words: stars: evolution - binaries: close - supernovae: general - white dwarfs

\section{INTRODUCTION}

Type Ia supernovae (SNe Ia) are among the most powerful explosions in the Universe and have high scientific values in Cosmology, e.g. they are used to determine the cosmological parameters due to their high luminosities and remarkable uniformity (for a recent review see Howell 2011). They are also a predominant synthesis of chemical elements in their host galaxies, especially for the contribution of iron (e.g. Matteucci \& Greggio 1986). It has been

\footnotetext{
* E-mail:wangbo@ynao.ac.cn
} 
accepted that SNe Ia arise from thermonuclear runaway explosions of carbon-oxygen white dwarfs (CO WDs) in binaries, although their progenitor systems and explosion mechanisms are still under debate (see, e.g. Podsiadlowski et al. 2008; Hillebrandt et al. 2013; Höflich et al. 2013; Wang et al. 2013; Zhang et al. 2016).

Over the past four decades, two main families of SN Ia progenitor scenarios have been discussed frequently. (1) The single-degenerate (SD) scenario (e.g. Whelan \& Iben 1973; Nomoto, Thielemann \& Yokoi 1984). In this scenario, a CO WD accretes hydrogen- or helium-rich matter from a non-degenerate star to increase its mass close to the Chandrasekhar limit and then results in a SN Ia explosion (e.g. Hachisu, Kato \& Nomoto 1996; Li \& van den Heuvel 1997; Han \& Podsiadlowski 2004; Meng, Chen \& Han 2009; Wang et al. 2009a; Lü et al. 2009; Chen \& Li 2009; Wu et al. 2016). (2) The double-degenerate (DD) scenario (e.g. Webbink 1984; Iben \& Tutukov 1984). In this scenario, a CO WD merges with another CO WD, the merging of which is due to the gravitational wave radiation that drives orbital inspiral to merger, resulting in a SN Ia explosion (e.g. Nelemans et al. 2001; Geier et al. 2007; Chen et al. 2012; Ji et al. 2013; Ruiter et al. 2013; Liu et al. 2016). Some variants of these two progenitor scenarios are needed to explain the observed diversity of SNe Ia (for recent reviews see, e.g. Wang \& Han 2012; Maoz, Mannucci \& Nelemans 2014; Ruiz-Lapuente 2014).

Most simulations and calculations for the DD scenario relate to the merger of two cold CO WDs 11 Meanwhile, a CO WD can also merge with the hot CO core of an asymptotic giant branch (AGB) star, and then produce a SN Ia, which is known as the core-degenerate (CD) scenario (e.g. Kashi \& Soker 2011; Ilkov \& Soker 2012; Soker 2013). The merger of a WD with the hot core of an AGB star was first investigated by Sparks \& Stecher (1974) who suggested that a SN could be formed through this type of merger. Livio \& Riess (2003) argued that the merger of the WD with the AGB core can explain the presence of hydrogen lines in some observed SNe Ia such as SN 2002ic when the merger happens at the end of the common-envelope (CE) stage or shortly after. Importantly, the CD scenario may avoid the off-center carbon ignition during the merger process (e.g. Soker 2013).

In the CD scenario, a SN Ia explosion may occur shortly or a long time after the CE stage (e.g. Soker et al. 2013). Kashi \& Soker (2011) suggested that this scenario could form massive WDs with super-Chandrasekhar mass, leading to the formation of super-luminous SNe Ia (see also Ilkov \& Soker 2012). In addition, a violent prompt merger via the CD scenario may reproduce the properties of some SNe Ia with circumstellar material (CSM) such as PTF $11 \mathrm{kx}$ (e.g. Soker et al. 2013). This scenario was also used to explain the properties of SN 2011fe and SN 2014J (e.g. Soker et al. 2014; Soker 2015). Aznar-Siguán et al. (2015) recently performed three-dimensional smoothed particle hydrodynamics simulations of the merger stage between the WD and the AGB core, and argued that this merge process can result in the formation of a massive WD and then produce a SN Ia. We note that Tsebrenko \& Soker (2015) recently summarized the properties of the CD scenario and made detailed comparisons between this scenario and other progenitor scenarios.

Although the CD scenario may explain some properties of SN Ia diversity, SN Ia birthrates from this scenario are still not well determined (e.g. Ilkov \& Soker 2013; Aznar-Siguán et al. 2015; Zhou et al. 2015). Ilkov \& Soker (2013) argued that this scenario can reproduce the observed birthrates of total SNe Ia based on a simplified binary population synthesis (BPS) code. Tsebrenko \& Soker (2015) recently estimated that more than $20 \%$ of all SNe Ia come from the CD scenario on the basis of the fraction of SNe Ia that happen inside

1 Note that the merger of two CO WDs may lead to an off-center carbon ignition, resulting in accretion induced collapse but not a SN Ia (e.g. Saio \& Nomoto 1985, 2004; Timmes, Woosley \& Taam 1994). 
planetary nebulae. Briggs et al. (2015) also carried out some BPS studies for the merger of WD+AGB core, and argued that the majority of magnetic WDs with strong fields are the carbon-oxygen type and may merge within a CE. Thus, Briggs et al. (2015) suggested that the merger of WD+AGB core may be relatively common.

The purpose of this article is to investigate SN Ia birthrates and delay times for the CD scenario using a detailed Monte Carlo BPS approach. We describe the numerical methods and assumptions for the BPS approach in Sect. 2, and give the BPS results in Sect. 3. Finally, a discussion and summary are presented in Sect. 4.

\section{NUMERICAL METHODS}

\subsection{Physical input}

In the CD scenario, a Chandrasekhar or super-Chandrasekhar mass WD could be formed through the merger of a cold CO WD with the hot CO core of an AGB star. A series of Monte Carlo BPS simulations for the CD scenario are performed in this study. We adopted the following assumptions as the criteria for producing SNe Ia through the CD scenario (e.g. Soker 2013; Ilkov \& Soker 2013): (1) The combined mass of the CO WD ( $M_{\mathrm{WD}}$, primary) and the AGB core ( $M_{\text {core }}$, secondary) during the final stage of CE evolution is larger than or equal to the Chandrasekhar mass limit $\left(1.378 M_{\odot}\right.$ in this work), that is, $M_{\mathrm{WD}}+M_{\text {core }} \geq 1.378 M_{\odot} 2$ (2) The WD and the AGB core merge during the final stage of CE evolution, in which the mass of the AGB core $\left(M_{\text {core }}\right)$ is limited to be lower than $1.1 M_{\odot}$ to avoid the formation of ONe cores that cannot produce SNe Ia.

The CO WD is usually disrupted and accreted onto the more massive AGB core during the merging process. However, in some conditions the AGB core would be disrupted and accreted onto the cooler CO WD if $M_{\mathrm{WD}}$ is larger than $M_{\text {core }}$ (e.g. Soker et al. 2013). In such case, the SN explosion may occur shortly after the CE stage, resulting in a SN Ia explosion inside a planetary nebula shell, which may reproduce the properties of some SNe Ia with CSM such as PTF $11 \mathrm{kx}$. This case is known as the violent prompt merger scenario (e.g. Soker et al. 2013), which is studied in the present work. Additionally, we also examined the influence of metallicity on the birthrates of SNe Ia for the CD scenario, in which metallicities were chosen to be $\mathrm{Z}=0.0001,0.004,0.02$ and 0.03 .

The delay times of SNe Ia are defined as the timescale from the formation of primordial binaries to SN explosions. In the CD scenario, the theoretical delay times of SNe Ia are the sum of the evolutionary timescale from primordial binaries to the formation of CD systems and the spin-down timescale from the merger product of WD+AGB core to SN explosion. The spin-down timescale of the merger product is mainly determined by the magneto-dipole radiation torque, which can be written as

$\tau_{\mathrm{B}} \approx 5 \times 10^{7}\left(\frac{B \sin \delta}{10^{7} \mathrm{G}}\right)^{-2} \mathrm{yr}$,

where $B \sin \delta$ follows a distribution of

$\frac{d N}{d \log (B \sin \delta)}=$ constant

for $10^{6} \mathrm{G} \leq B \sin \delta \leq 10^{8} \mathrm{G}$ (e.g. Ilkov \& Soker 2012; Meng et al. 2012). According to this

2 Nomoto, Thielemann \& Yokoi (1984) suggested that the critical mass limit of a cold non-rotating WD for carbon ignition is about $1.378 M_{\odot}$. Note that the Chandrasekhar mass limit for WDs is $1.44 M_{\odot}$ in Newtonian gravity, which drops to $1.4 M_{\odot}$ when general relativity is taken into account (see Mathew \& Nandy 2014). 
distribution, we can get the spin-down timescale using a Monte Carlo simulations. Note that there is no time delay by spin-down or gravitational wave radiation for the case of these violent prompt mergers (e.g. Soker et al. 2013), and the spin-down timescale can be neglected for those with merging masses above $1.48 M_{\odot}$ in our simulations. The massive WDs over $1.48 M_{\odot}$ require differential rotation for support before explosions (e. g. Yoon \& Langer 2004). The spin-down time of these WDs is mainly determined by the time-scale of internal angular-momentum redistribution, which may be extremely short as the angular momentum transport by the Eddington-Sweet meridional circulation is relatively fast (see, e.g. Yoon \& Langer 2004; Saio \& Nomoto 2004; Piro 2008; Hachisu et al. 2012).

\subsection{Basic parameters for Monte Carlo simulations}

We carried out a series of Monte Carlo BPS simulations for the CD scenario. In each simulation, we followed the evolution of $1 \times 10^{7}$ primordial binaries from star formation to the formation of WD+AGB systems using the Hurley binary evolution code (see Hurley, Tout \& Pols 2002). We simply supposed a constant star formation rate (SFR) of $5 M_{\odot} \mathrm{yr}^{-1}$ over the past $14 \mathrm{Gyr}$ or, alternatively, it is supposed as a delta function, i.e. a single instantaneous starburst. The constant SFR is used to approximate spiral galaxies, whereas the delta function provides a rough description of globular clusters or elliptical galaxies.

The Monte Carlo BPS simulations require as input the initial mass function (IMF) of the primordial stars, the initial mass-ratio distribution, the distribution of initial orbital separations, and the eccentricity distribution of binary orbit. The Monte Carlo BPS studies are highly dependent on the chosen initial conditions. The following initial parameters for the Monte Carlo simulations are adopted: (1) The initial mass function (IMF) for the primordial primary is taken from Miller \& Scalo (1979, MS79). Alternatively, we also consider the IMF of Scalo (1986, S86). (2) A constant mass-ratio distribution is supposed (e.g. Mazeh et al. 1992; Goldberg \& Mazeh 1994). Alternatively, we also adopt an uncorrelated mass-ratio distribution, in which both binary components are chosen independently from the same IMF. (3) The distribution of initial orbital separations is supposed to be constant in $\log a$ for wide binaries, in which $a$ is the orbital separation (for more discussions see Eggleton, Fitchett \& Tout 1989; see also Han et al. 1995). (4) All stars are supposed to be members of binaries. The primordial binaries are generated through a Monte Carlo method, and a circular orbit is supposed for all binaries. It is usually not necessary to model an initial eccentricity distribution of binary orbit in BPS studies as binary systems tend to circularise before interacting (see Hurley, Tout \& Pols 2002).

\subsection{Evolutionary way to WD+AGB systems}

SN Ia explosions in the CD scenario originate from the evolution of WD+AGB systems. In Fig. 1, we present the binary evolutionary way to form WD+AGB systems. The primordial binary system in the CD scenario has a wide separation, which allows the primordial primary to evolve into the AGB stage. The primordial primary first fills its Roche lobe when it evolves to AGB stage (it now contains a $\mathrm{CO}$ core in its center). Before the primordial primary fills its Roche-lobe, it loses a lot of matter through the stellar wind in the AGB stage, which leads to the subsequent stable Roche-lobe overflow (RLOF). In the Hurley binary evolution code, the treatment of RLOF is a revised version originated from Tout et al. (1997), and the radius-mass exponent $\zeta$ defined by Webbink (1985) is employed to deal with the stability of mass transfer. At the end of RLOF, the primary become a CO WD. Meanwhile, the MS secondary become a massive star due to the stable mass transfer, resulting in the formation of 


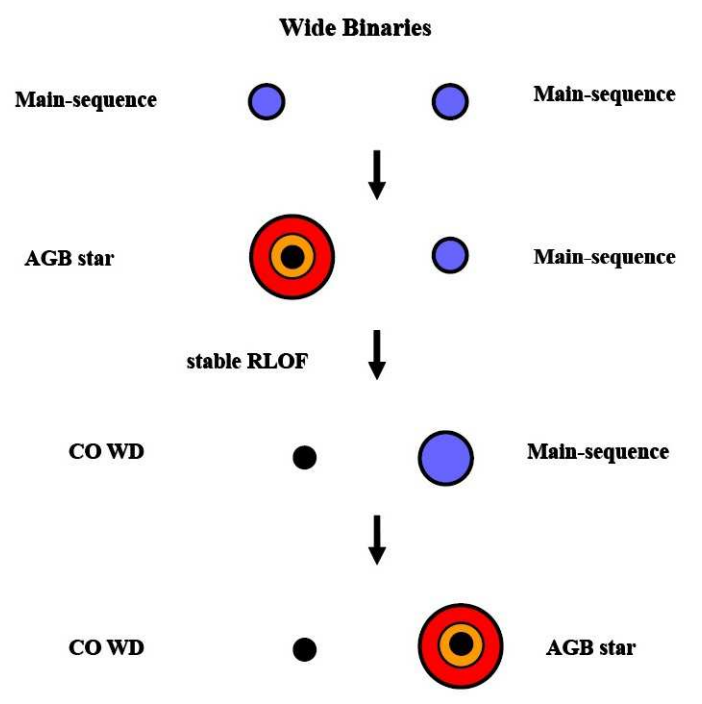

Figure 1. Binary evolutionary way to WD+AGB systems.

a CO WD+MS system. The CO WD+MS system continues to evolve, and the MS secondary may fill its Roche-lobe again when it evolves to the AGB stage. At this stage, a CE may be formed due to the dynamically unstable mass transfer, resulting from the deep convective envelope of the AGB star and the large mass-ratio. The CO WD will merge with the CO core of the AGB star during the CE stage if the CE cannot be ejected. Finally, a SN Ia is produced at the final stage of the CE evolution (see also Ilkov \& Soker 2013).

For the CD scenario, SN Ia explosions for the ranges of the initial mass of the primordial primary are from 2.0-6.5 $M_{\odot}$, and the initial mass of the primordial secondary from 1.5-6.0 $M_{\odot}$. The initial orbital period of the primordial system needs to be wide enough (e.g. larger than $8 \mathrm{yr}$ ) so that the primordial primary can evolve to an AGB star before it fills its Roche-lobe.

\subsection{Common envelope evolution}

In the $\mathrm{CD}$ scenario, the merger of $\mathrm{WD}+\mathrm{AGB}$ core originates from the $\mathrm{CE}$ evolution. In oder to obtain the output of the CE stage, the standard energy equation is adopted (e.g. Webbink 1984). The CE is supposed to be ejected once

$\alpha_{\mathrm{CE}}\left(\frac{G M_{\mathrm{don}}^{\mathrm{f}} M_{\mathrm{acc}}}{2 a_{\mathrm{f}}}-\frac{G M_{\mathrm{don}}^{\mathrm{i}} M_{\mathrm{acc}}}{2 a_{\mathrm{i}}}\right)=\frac{G M_{\mathrm{don}}^{\mathrm{i}} M_{\mathrm{env}}}{\lambda R_{\mathrm{don}}}$,

where $G$ is the gravitational constant, $\alpha_{\mathrm{CE}}$ is the $\mathrm{CE}$ ejection efficiency, $M_{\text {acc }}$ is the mass of the accreting star, $M_{\text {don }}$ is the mass of the donor, $a$ is the orbital separation, $R_{\text {don }}$ is the radius of the donor, $M_{\text {env }}$ is the envelope mass of the donor, $\lambda$ is a structure parameter of the donor relevant to the stellar mass-density distribution, and the indices $\mathrm{i}$ and $\mathrm{f}$ indicate the initial and final values during the CE stage. The left side of this equation presents the difference of orbital energy between the final and initial stage, whereas the right side gives the binding energy of the CE.

There are two highly uncertain parameters in equation (3), that is, $\alpha_{\mathrm{CE}}$ and $\lambda$, which are key parts of our understanding the orbital evolution of the binary during the CE stage. Previous studies usually combined $\alpha_{\mathrm{CE}}$ and $\lambda$ into a single free parameter $\alpha_{\mathrm{CE}} \lambda$ when calculating the output of CE stage (e.g. Wang et al. 2009b). The value of $\lambda$ depends on the 
Table 1. Galactic SN Ia birthrates for different Monte Carlo BPS simulation sets. Notes: $\alpha_{\mathrm{CE}}=\mathrm{CE}$ ejection efficiency; $\alpha_{\mathrm{in}}$ $=$ percentage of the internal energy contributing to the ejection of the envelope; IMF = initial mass function; $n(q)=$ initial mass-ratio distribution; $\nu_{\mathrm{CD}}\left(\nu_{\mathrm{CSM}}\right)=\mathrm{SN}$ Ia birthrates for the CD scenario (SNe Ia with CSM); $\nu_{\mathrm{SD}}=\mathrm{SN}$ Ia birthrates for the SD scenario that includes the contributions of WD+MS/RG channels based on the studies of Wang, Li \& Han $(2010) ; \nu_{\mathrm{DD}}=$ SN Ia birthrates for the DD scenario based on the studies of Wang et al. (2010).

\begin{tabular}{cccccccc}
\hline \hline Set & $\alpha_{\mathrm{CE}}$ & $\alpha_{\mathrm{in}}$ & $\mathrm{IMF}$ & $n(q)$ & $\begin{array}{c}\nu_{\mathrm{CD}}\left(\nu_{\mathrm{CSM}}\right) \\
\left(10^{-3} \mathrm{yr}^{-1}\right)\end{array}$ & $\begin{array}{c}\nu_{\mathrm{SD}} \\
\left(10^{-3} \mathrm{yr}^{-1}\right)\end{array}$ & $\begin{array}{c}\nu_{\mathrm{DD}} \\
\left(10^{-3} \mathrm{yr}^{-1}\right)\end{array}$ \\
\hline 1 & 0.1 & 1 & MS79 & Constant & $0.852(0.566)$ & 1.768 & 0.707 \\
2 & 0.2 & 1 & MS79 & Constant & $0.440(0.323)$ & 1.480 & 0.158 \\
3 & 0.2 & 0 & MS79 & Constant & $0.479(0.295)$ & 0.899 & 0.358 \\
4 & 0.2 & 1 & S 86 & Constant & $0.373(0.279)$ & 1.072 & 0.133 \\
5 & 0.2 & 1 & MS79 & Uncorrelated & $0.034(0.029)$ & 0.257 & 0.011 \\
6 & 0.3 & 1 & MS79 & Constant & $0.116(0.090)$ & 1.221 & 0.077 \\
7 & 0.5 & 1 & MS79 & Constant & $0.050(0.041)$ & 1.329 & 0.100 \\
\hline
\end{tabular}

structure and evolutionary stage of the donor, which is usually set to be 0.5 for simplicity (e.g. Hurley et al. 2002). However, the real value of $\lambda$ may be far from 0.5 (e.g. van der Sluys, Verbunt \& Pols 2006; see also Dewi \& Tauris 2000). In this paper, we used the fitting formulae of envelope binding energy $E_{\text {bind }}$ of the original giant, which implicitly contain the variable $\lambda$ (see Loveridge, van der Sluys \& Kalogera 2011). The $E_{\text {bind }}$ is estimated by integrating the gravitational and internal energies from the core-envelope boundary $\left(M_{\mathrm{c}}\right)$ to the surface of the star $\left(M_{\mathrm{s}}\right)$,

$E_{\mathrm{bind}}=\int_{M_{\mathrm{c}}}^{M_{\mathrm{s}}}\left(-\frac{G m}{r(m)}\right) d m+\alpha_{\mathrm{in}} \int_{M_{\mathrm{c}}}^{M_{\mathrm{s}}} E_{\mathrm{in}} d m$,

in which $\alpha_{\text {in }}$ is the percentage of the internal energy contributing to the ejection of the envelope and $E_{\text {in }}$ is the internal energy including the thermal energy of the gas and the radiation energy (e.g. van der Sluys, Verbunt \& Pols 2006; Zuo \& Li 2014). $\alpha_{\text {in }}$ is usually set to be 1 though its value is rather uncertain. Here, we take $\alpha_{\text {in }}$ as 1 in our basic model and change it to zero for comparisons (see set 3 in Table 1). Meanwhile, we also change the values of $\alpha_{\mathrm{CE}}$ (e.g. 0.1, 0.2, 0.3 and 0.5 ) to examine its influence on the final results.

\section{RESULTS}

\subsection{Birthrates of SNe Ia}

The observed SN Ia birthrate in our Galaxy is about $4 \times 10^{-3} \mathrm{yr}^{-1}$, which can be used to constrain the progenitor models of SNe Ia (e.g. Cappellaro \& Turatto 1997). In order to systematically study the Galactic SN Ia birthrates for the CD scenario, we carried out seven sets of Monte Carlo BPS simulations with metallicity $Z=0.02$ (see Table 1 ). In these simulations, we varied the initial input parameters to examine their influences on the BPS results. For comparisons, we also show the results of SNe Ia for the SD and DD scenarios in this table. We found that the birthrates of SNe Ia are sensitive to uncertainties in some model parameters based on the seven sets of simulations. For example, if we adopt a mass-ratio distribution with uncorrelated binary components, the birthrates of SNe Ia will decrease significantly (set 5).

In Fig. 2, we show the evolution of the Galactic SN Ia birthrates with time for the CD scenario by adopting metallicity $Z=0.02$ and a constant SFR of $5 M_{\odot} \mathrm{yr}^{-1}$. According to the seven sets of Monte Carlo simulations, the theoretical birthrates from this scenario are in the range of $0.5-8.5 \times 10^{-4} \mathrm{yr}^{-1}$, accounting for $1-20 \%$ of the observations. The SN Ia birthrates from the CD scenario are lower than those in observations, which means that the 


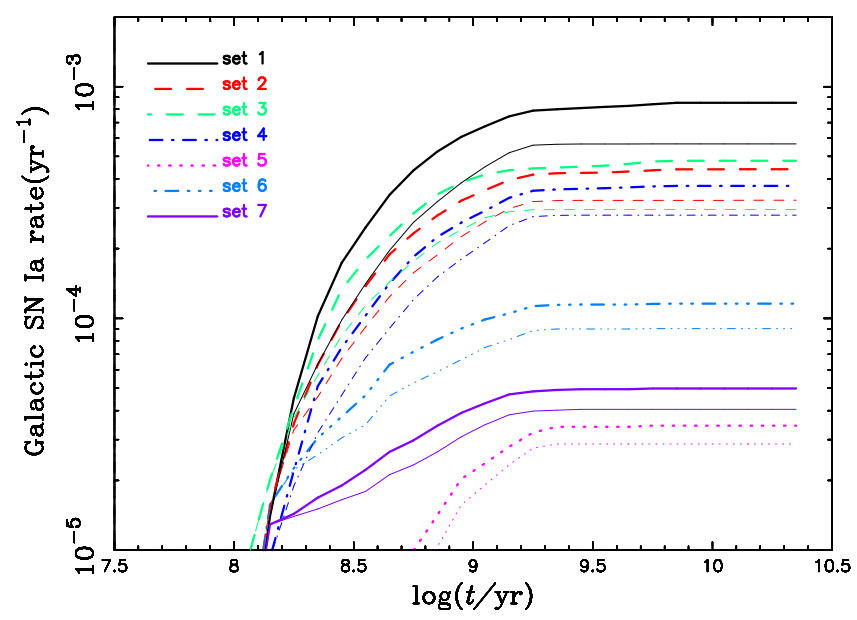

Figure 2. Evolution of the Galactic SN Ia birthrates with time for a constant Population I SFR with different BPS simulation sets. The thick lines are for all SNe Ia from the CD scenario, whereas the thin lines are only for the SNe Ia with CSM like PTF $11 \mathrm{kx}$.

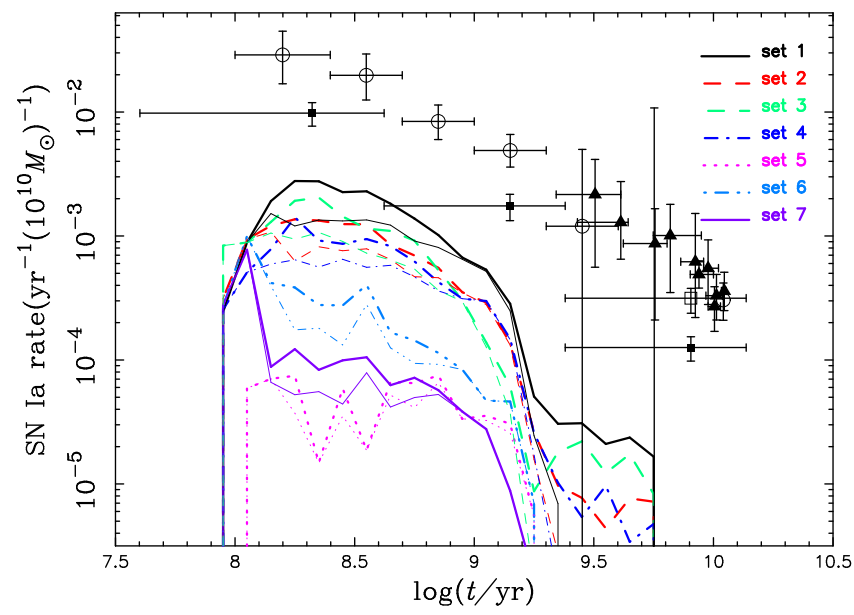

Figure 3. Delay-time distributions of SNe Ia with different BPS simulation sets, where the spin-down time is included in the delay time. The thick lines are for all SNe Ia from the CD scenario, whereas the thin lines are only for SNe Ia with CSM like PTF 11kx. The open circles are taken from Totani et al. (2008), the open square is from Graur \& Maoz (2013), the filled triangles and squares are from Maoz, Keren \& Avishay (2010) and Maoz, Mannucci \& Timothy (2012), respectively.

CD scenario can only form part of total SNe Ia. We note that the birthrates of SNe Ia from the CD scenario fall with increasing the value of $\alpha_{\mathrm{CE}}$. This is because a high value of $\alpha_{\mathrm{CE}}$ leads to wider orbital separation, resulting in few mergers of WD+AGB core. If the CD scenario can really contribute to a high proportion of observed SNe Ia, a low value of $\alpha_{\mathrm{CE}}$ is expected.

Soker et al. (2013) recently suggested that the violent prompt merger in the CD scenario may account for the very massive CSM in PTF $11 \mathrm{kx}$, and for the presence of hydrogen shells in the CSM. According to our BPS simulations, the Galactic birthrates for SNe Ia with CSM are in the range of $0.3-5.7 \times 10^{-4} \mathrm{yr}^{-1}$, accounting for $0.7-10 \%$ of total SNe Ia, which can reproduce the birthrates of SNe Ia like PTF 11kx; the observed fraction of SNe Ia with CSM is estimated to be $0.1-1 \%$ (e.g. Dilday et al. 2012).

\subsection{Delay times of SNe Ia}

The delay time distributions of SNe Ia can be obtained from observations, which could be used to constrain the progenitor models of SNe Ia. In Fig. 3, we present the SN Ia delay 


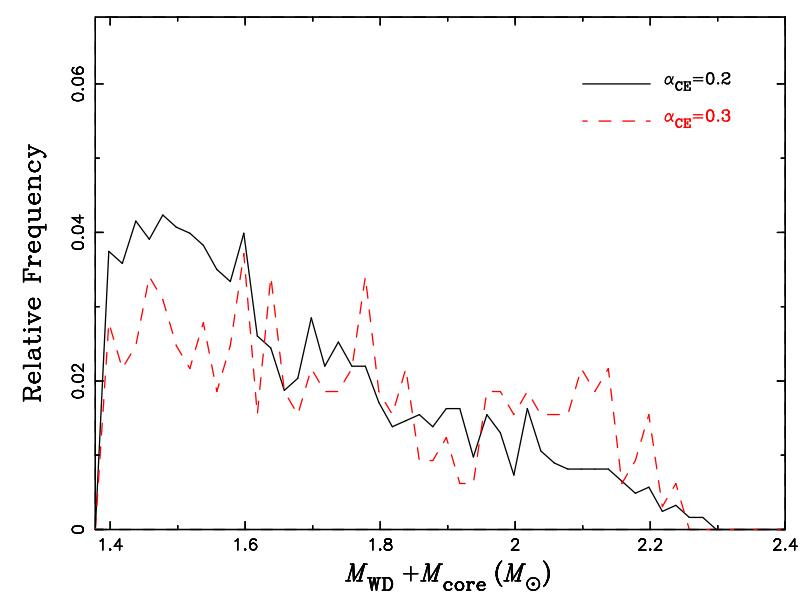

Figure 4. Distribution of the total mass of $M_{\mathrm{WD}}$ and $M_{\text {core }}$ in the WD+AGB systems that can ultimately form SNe Ia. The black solid and red dashed lines present the cases of $\alpha_{\mathrm{CE}}=0.2$ (set 2) and $\alpha_{\mathrm{CE}}=0.3$ (set 6), respectively. The number in every case is normalized to 1 .

time distributions for the CD scenario based on a single starburst of $10^{10} M_{\odot}$, where the spin-down time is included in the delay time. The estimated delay times for this scenario are mainly in the range of $90 \mathrm{Myr}-2500 \mathrm{Myr}$ after the starburst, which may have a contribution to the SNe Ia with short and intermediate delay times. For the SNe Ia with CSM, the SN explosion may occur shortly after the CE stage. The delay time of SNe Ia in this case is mainly determined by the evolutionary timescale from primordial binaries to the formation of CD systems. Note that the shortest delay times of SNe Ia for the CD scenario are mainly determined by the MS lifetime of a star with mass about $7 M_{\odot}$.

As expected in some previous studies (e.g. Kashi \& Soker 2011; Ilkov \& Soker 2012), we also found that the CD scenario can even form some SNe Ia with delay times as long as several Gyr for a low value of $\alpha_{\mathrm{CE}}$ (e.g. $\alpha_{\mathrm{CE}}=0.1$ and 0.2 ); a low value of $\alpha_{\mathrm{CE}}$ tends to form more mergers with masses below $1.48 M_{\odot}$, and thus a long spin-down time. The delay time of SNe Ia in this case is mainly determined by the spin-down time after the merging of the WD+AGB core. If the spin-down time is too long (e.g. several Gyr), the mergers may experience post-crystallization stage, leading to the formation of a highly carbon enriched outer layer. Thus, Soker et al. (2013) suggested that this case may account for the carbon-rich composition of the fastest-moving ejecta of SN $2011 \mathrm{fe}$.

\subsection{Mass distribution of WD+AGB core}

The WD explosion mass may have an influence on the final production of nickel-56 and thus the maximum luminosity of SNe Ia (e.g. Arnett 1982). In Fig. 4, we show the distribution of the combined masses of $M_{\mathrm{WD}}$ and $M_{\text {core }}$ in the WD+AGB systems that can ultimately form SNe Ia through the CD scenario. The combined masses have a peak nearby $1.4 M_{\odot}$ and then decrease, which can be understood by the initial mass function of stars. These massive systems with combined masses above $2 M_{\odot}$ may contribute to over-luminous SNe Ia that have been observed with inferred WD explosion masses in the order of $2 M_{\odot}$ (e.g. Howell et al. 2006; Hicken et al. 2007; Scalzo et al. 2010). Note that the SD scenario of SNe Ia can also form over-luminous SNe Ia if the rotation of WDs is considered, in which the rotating WDs have been prevented from exploding until angular-momentum redistribution (e.g. Justham 2011; Hachisu et al. 2012; Wang et al. 2014).

Fig. 5 presents the distribution of mass ratio between $M_{\mathrm{WD}}$ and $M_{\text {core }}$ with different values of $\alpha_{\mathrm{CE}}$. From this figure, we can see that almost all the values of $M_{\mathrm{WD}} / M_{\text {core }}$ are 


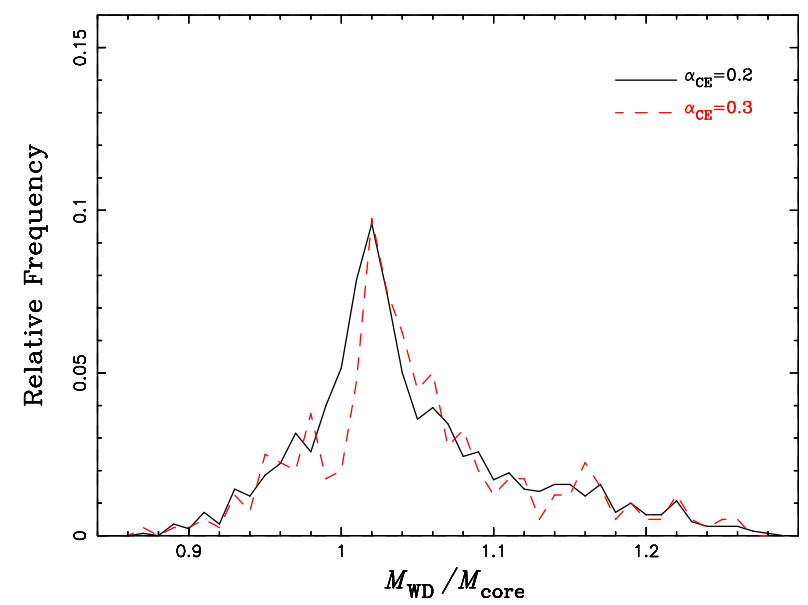

Figure 5. Same as Fig. 4, but for the distribution of mass ratio between $M_{\mathrm{WD}}$ and $M_{\text {core }}$.

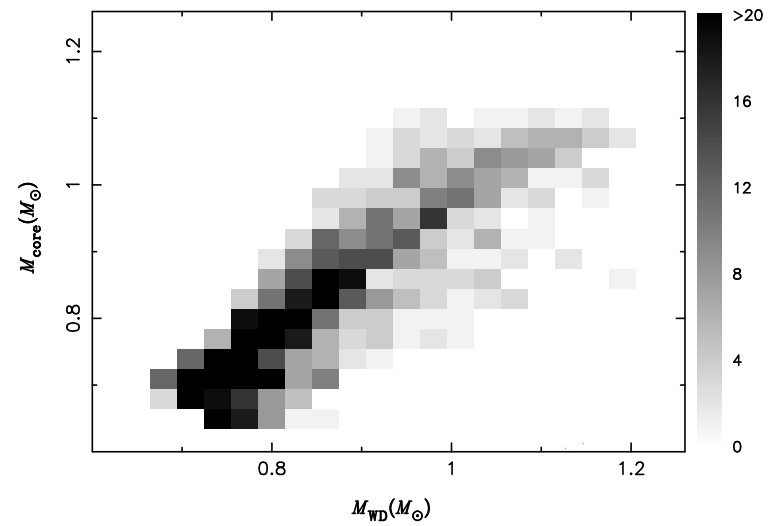

Figure 6. Distribution of $M_{\mathrm{WD}}$ and $M_{\mathrm{core}}$ in WD+AGB systems that can ultimately form SNe Ia, where $\alpha_{\mathrm{CE}}=0.2$ (set 2).

above 0.9 , and there is a peak nearby 1.0. In Fig. 6, we show the distribution of $M_{\mathrm{WD}}$ and $M_{\text {core }}$ in the WD+AGB systems that can ultimately form SNe Ia. In this figure, WD+AGB systems with $M_{\text {core }}>1.1 M_{\odot}$ are rejected as more massive CO cores in AGB stars have already burnt all their central carbon and will form ONe cores (e.g. Umeda et al. 1999).

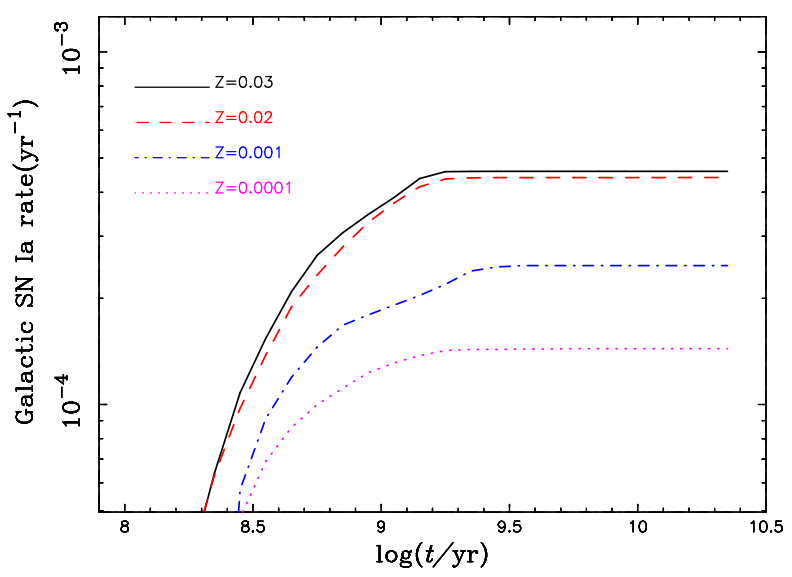

Figure 7. The evolution of SN Ia birthrates for a constant SFR with different metallicities of $Z=0.03,0.02,0.001$ and 0.0001 , where $\alpha_{\mathrm{CE}}=0.2$. 


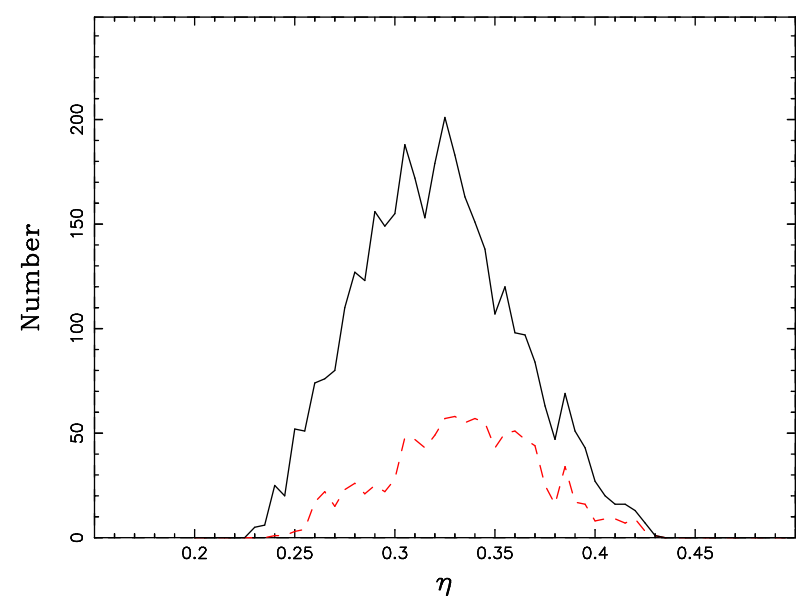

Figure 8. Distribution of the mass-transfer parameter $\eta$ in our simulations, where $\alpha_{\mathrm{CE}}=0.2$ (set 2). The black solid line presents the distribution of $\eta$ for all potential WD+AGB systems, whereas the red dashed line shows the distribution of $\eta$ for these WD+AGB systems that can lead to SNe Ia.

\subsection{The effect of metallicity}

Metallicities may have important effects on the properties of SNe Ia (e.g. Timmes, Brown \& Truran 2003; Podsiadlowski et al. 2008; Sullivan et al. 2010). In Fig. 7, we present the evolution of SN Ia birthrates for a constant SFR of $5 M_{\odot} \mathrm{yr}^{-1}$ with different metallicities. From this figure, we can see that metallicity has a significant influence on the birthrates of SNe Ia, especially for low metallicity environments; the birthrates increase with metallicity and SNe Ia happen systemically earlier for a high value of metallicity. The WD+AGB systems mainly originate from massive primordial binaries that tend to exist in high metallicity environments (e.g. Wang \& Han 2010). Thus, SN Ia birthrates from the CD scenario increase with metallicity. Meanwhile, massive primordial binaries evolve more quickly than low-mass ones, resulting in a systematically earlier SN explosion for a high value of metallicity.

\section{DISCUSSION AND SUMMARY}

According to a simple BPS code, Ilkov \& Soker (2013) suggested that the CD scenario can reproduce the observed birthrates of SNe Ia, and claimed that this scenario plays an important role for producing SNe Ia. However, we found that this scenario can only account for part (1-20\%) of SNe Ia based on our detailed BPS approach. The main difference between these two works is the treatment of the binary interaction during mass transfer. Ilkov \& Soker (2013) obtained the new mass of the primordial secondary after the primordial primary passed through the AGB stage and became a WD (with mass $M_{\mathrm{WD}}$ ) as $M_{2 \text { new }}=$ $M_{2}+\eta\left(M_{1}-M_{\mathrm{WD}}\right)$, where $\eta$ is the mass-transfer parameter, $M_{1}$ and $M_{2}$ are the initial masses of the primordial primary and secondary, respectively. In Fig. 8, we show the distribution of the mass-transfer parameter $\eta$ in our simulations. From this figure, we can see that the value of $\eta$ in our simulations is mainly in the range of $0.25-0.4$ that is much lower than the value ( $\eta \sim 0.8-0.9)$ taken by Ilkov \& Soker (2013). The high mass-transfer parameter in Ilkov \& Soker (2013) leads to the formation of more massive AGB stars and larger CO cores, and thus gives a much higher birthrates of SNe Ia.

In this work, we assume that SNe Ia in the CD scenario can be produced during the final stage of $\mathrm{CE}$ evolution. However, a SN Ia can also be formed through the CD scenario when the merging process of double WDs happens shortly after the CE stage (e.g. within about $10^{5} \mathrm{yr}$ ), the merging of which is due to the gravitational wave radiation (e.g. Soker 
2013). Meng \& Yang (2012) recently estimated that SN Ia birthrates from this case is very low $(<0.1 \%$ to all SNe Ia). Thus, we speculate that the condition studied here dominates the production of SNe Ia in the CD scenario although this scenario only contributes to part of the observed SNe Ia.

Soker et al. (2013) recently suggested that the violent prompt merger in the CD scenario may explain some SNe Ia with very massive CSM such as PTF $11 \mathrm{kx}$. If we adopted a strict assumption on the total mass of the WD+AGB core and the envelope mass $\left(M_{\text {env }}\right)$ of the AGB star (e.g. $M_{\mathrm{WD}}+M_{\text {core }} \geq 1.8 M_{\odot}$ and $M_{\mathrm{env}} \geq 0.5 M_{\odot}$; Soker et al. 2013), the SN Ia birthrates from the violent prompt merger scenario will decrease to $0.3-8.0 \times 10^{-5} \mathrm{yr}^{-1}$, accounting for $0.1-2 \%$ of all SNe Ia, which can still reproduce the observational number of SNe Ia like PTF $11 \mathrm{kx}$.

By employing a detailed BPS approach, we got an upper limit for the birthrates of SNe Ia based on the CD scenario (no more than $20 \%$ of total SNe Ia). The birthrates in our simulations are lower than those in Ilkov \& Soker (2013), the main reason of which is that we adopted a detailed mass-transfer process. We found that the CD scenario mainly contributes to the SNe Ia with short and intermediate delay times although this scenario can also produce some SNe Ia with long delay times. The birthrates of SNe Ia with CSM are estimated to be $0.7-10 \%$ of total SNe Ia, which can match the observed number of SNe Ia like PTF $11 \mathrm{kx}$. We also found that SNe Ia happen systemically earlier for a high value of metallicity and their birthrates increase with metallicity. In order to put further constraints on the CD scenario, more numerical simulations and observational evidence for this scenario are needed.

\section{ACKNOWLEDGMENTS}

We acknowledge the referee, Christopher Tout, for the valuable comments that helped us to improve the paper. This work is supported by the National Basic Research Program of China (973 programme, 2014CB845700), the National Natural Science Foundation of China (Nos 11673059, 11322327, 11390374, 11521303, 11303036, 11573021, 11403096 and 61561053), the Chinese Academy of Sciences (Nos QYZDB-SSW-SYS001, XDB09010202 and KJZD-EWM06-01), the Fundamental Research Funds for the Central Universities, the Natural Science Basic Research Program of Shaanxi Province - Youth Talent Project (No. 2016JQ1016), the Natural Science Foundation of Yunnan Province (Nos 2013HB097 and 2013FB083), and the Youth Innovation Promotion Association CAS.

\section{REFERENCES}

Arnett W. D., 1982, ApJ, 253, 785

Aznar-Siguán G., García-Berro E., Lorén-Aguilar P., Soker N., Kashi A., 2015, MNRAS, 450, 2948

Briggs G. P. et al., 2015, MNRAS, 447, 1713

Cappellaro E., Turatto M., 1997, In: Ruiz-Lapuente P., Cannal R., Isern J. (Eds.), Proceedings of the NATO Advanced Study Institute, Thermonuclear Supernovae, Vol. 486.

Kluwer, Dordrecht, p. 77

Chen W.-C., Li X.-D., 2009, ApJ, 702, 686

Chen X., Jeffery C. S., Zhang X., Han Z., 2012, ApJ, 755, L9

Dewi J. D. M., Tauris T. M., 2000, A\&A, 360, 1043

Dilday B. et al., 2012, Science, 337, 942 
Eggleton P. P., Fitchett M. J., Tout C. A., 1989, ApJ, 347, 998

Geier S., Nesslinger S., Heber U., Przybilla N., Napiwotzki R., Kudritzki R. P., 2007, A\&A, 464, 299

Graur O., Maoz D., 2013, MNRAS, 430, 1746

Hachisu I., Kato M., Nomoto K., 1996, ApJL, 470, L97

Hachisu I., Kato M., Saio H., Nomoto K., 2012, ApJ, 744, 69

Han Z., Podsiadlowski P., 2004, MNRAS, 350, 1301

Han Z., Podsiadlowski P., Eggleton P. P., 1995, MNRAS, 272, 800

Hicken M. et al., 2007, ApJ, 669, L17

Hillebrandt W., Kromer M., Röpke F. K., Ruiter A. J., 2013, FrPhy, 8, 116

Höflich P. et al., 2013, FrPhy, 8, 144

Howell D. A., 2011, Nature Communications, 2, 350

Howell D. A. et al., 2006, Nature, 443, 308

Hurley J. R., Tout C. A., Pols O. R., 2002, MNRAS, 329, 897

Iben I., Tutukov A. V., 1984, ApJS, 54, 335

Ilkov M., Soker N., 2012, MNRAS, 419, 1695

Ilkov M., Soker N., 2013, MNRAS, 428, 579

Ji S. et al., 2013, ApJ, 773, 136

Justham S., 2011, ApJ, 730, L34

Kashi A., Soker N., 2011, MNRAS, 417, 1466

Li X.-D., van den Heuvel E. P. J., 1997, A\&A, 322, L9

Liu D.,Wang B., Podsiadlowski P., Han Z., 2016, MNRAS, 461, 3653

Livio M., Riess A., 2003, ApJ, 594, L93

Loveridge A. J., van der Sluys M. V., Kalogera V., ApJ, 2011, 743, 49

Lü G., Zhu C., Wang Z., Wang N., 2009, MNRAS, 396, 1086

Maoz D., Mannucci F., Nelemans G., 2014, ARA\&A, 52, 107

Maoz D., Keren S., Avishay G.-Y., 2010, ApJ, 722, 1879

Maoz D., Mannucci F., Timothy D. B., 2012, MNRAS, 426, 3282

Mathew A., Nandy M. K., 2014, preprint (arXiv:1401.0819)

Matteucci F., Greggio L., 1986, A\&A, 154, 279

Meng X., Chen X., Han Z., 2009, MNRAS, 395, 2103

Meng X., Yang W., 2012, A\&A, 543, A137

Miller G. E., Scalo J. M., 1979, ApJS, 41, 513 (MS79)

Nelemans G., Yungelson L. R., Portegies Zwart S. F., Verbunt F., 2001, A\&A, 365, 491

Nomoto K., Thielemann F.-K., Yokoi K., 1984, ApJ, 286, 644

Piro A. L., 2008, ApJ, 679, 616

Podsiadlowski P., Mazzali P., Lesaffre P., Han Z., Förster F., 2008, New Astron. Rev., 52, 381

Ruiter A. J. et al., 2013, MNRAS, 429, 1425

Ruiz-Lapuente P., 2014, New Astron. Rev., 62, 15

Saio H., Nomoto K., 1985, A\&A, 150, L21

Saio H., Nomoto K., 2004, ApJ, 615, 444

Scalo J. M., 1986, Fund. Cosm. Phys., 11, 1 (S86)

Scalzo R. A. et al., 2010, ApJ, 713, 1073

Soker N., 2013, IAUS, 281, 72

Soker N., 2015, MNRAS, 450, 1333

Soker N. et al., 2013, MNRAS, 431, 1541

Soker N. et al., 2014, MNRAS, 437, L66

Sparks W. M., Stecher T. P., 1974, ApJ, 188, 149 
Sullivan M. et al., 2010, MNRAS, 406, 782

Timmes F. X., Woosley S. E., Taam R. E., 1994, ApJ, 420, 348

Timmes F. X., Brown E. F., Truran J. W., 2003, ApJ, 590, L83

Totani T., Morokuma T., Oda T., Doi M., Yasuda N., 2008, PASJ, 60, 1327

Tout C. A., Aarseth S. J., Pols O. R., Eggleton P. P., 1997, MNRAS, 291, 732

Tsebrenko D., Soker N., 2015, MNRAS, 447, 2568

Umeda H., Nomoto K., Yamaoka H., Wanajo S., 1999, ApJ, 513, 861

van der Sluys M. V., Verbunt F., Pols O. R., 2006, A\&A, 460, 209

Wang B., Meng X., Chen X., Han Z., 2009a, MNRAS, 395, 847

Wang B., Chen X., Meng X., Han Z., 2009b, ApJ, 701, 1540

Wang B. et al., 2010, Sci. China Ser. G, 53, 586

Wang B., Han Z., 2010, A\&A, 515, A88

Wang B., Han Z., 2012, New Astron. Rev., 56, 122

Wang B., Justham S., Liu Z., Zhang J., Liu D., Han Z., 2014, MNRAS, 445, 2340

Wang B., Li X.-D., Han Z., 2010, MNRAS, 401, 2729

Wang X.-F., Wang L., Filippenko A. V., Zhang T., Zhao X., 2013, Science, 340, 170

Webbink R., 1984, ApJ, 277, 355

Webbink R., 1985, in Interacting Binary Stars, eds. J. E. Pringle, \& R. A. Wade (Cambridge University Press), 39

Whelan J., Iben I., 1973, ApJ, 186, 1007

Wu C., Liu D., Zhou W., Wang B., 2016, RAA, 16, 160

Yoon S.-C., Langer N., 2004, A\&A, 419, 623

Zhang J.-J. et al., 2016, ApJ, 817, 114

Zhou W., Wang B., Meng X., Liu D., Zhao G., 2015, RAA, 15, 1701

Zuo Z.-Y., Li X.-D., 2014, MNRAS, 442, 1980 